University of Wollongong

Research Online

Faculty of Informatics - Papers (Archive)

Faculty of Engineering and Information

Sciences

$1-1-2012$

\title{
A fundamental analysis of continuous flow bioreactor and membrane reactor models with tessier kinetics
}

\author{
MI. Nelson \\ University of Wollongong, mnelson@uow.edu.au \\ E Balakrishnan \\ Sultan Qaboos University, balak@squ.edu.pm \\ H S. Sidhu \\ UNSW at ADFA, h.sidhu@adfa.edu.au
}

Follow this and additional works at: https://ro.uow.edu.au/infopapers

Part of the Physical Sciences and Mathematics Commons

\section{Recommended Citation}

Nelson, M I.; Balakrishnan, E; and Sidhu, H S.: A fundamental analysis of continuous flow bioreactor and membrane reactor models with tessier kinetics 2012, 417-433.

https://ro.uow.edu.au/infopapers/1874

Research Online is the open access institutional repository for the University of Wollongong. For further information contact the UOW Library: research-pubs@uow.edu.au 


\title{
A fundamental analysis of continuous flow bioreactor and membrane reactor models with tessier kinetics
}

\begin{abstract}
In this research we analyze the steady-state operation of a continuous flow bioreactor, with or without recycle, and an idealized or nonidealized continuous flow membrane reactor. The model extends to include a fixed bed reactor where a fraction of the biomass is detached by the flow. The reaction is assumed to be governed by Tessier growth kinetics. We show that a flow reactor with idealized recycle has the same performance as an idealized membrane reactor and that the performance of a nonidealized membrane reactor is identical to that of an appropriately defined continuous flow bioreactor with nonidealized recycle. The performance of all three reactor types can therefore be obtained by analyzing a flow reactor with recycle. The steady states of the recycle model are found and their stability determined as a function of the residence time. The performance of the reactor at large residence times is obtained.
\end{abstract}

\section{Keywords}

bioreactor, flow, continuous, analysis, fundamental, reactor, kinetics, tessier, membrane, models

\section{Disciplines}

Physical Sciences and Mathematics

\section{Publication Details}

Nelson, M. I., Balakrishnan, E. \& Sidhu, H. S. (2012). A fundamental analysis of continuous flow bioreactor and membrane reactor models with tessier kinetics. Chemical Engineering Communications, 199 (3), 417-433. 


\title{
A fundamental analysis of continuous flow bioreactor and membrane reactor models with Tessier kinetics
}

\author{
M.I. Nelson*(1), E. Balakrishnan (2), H.S. Sidhu (3) \\ (1) School of Mathematics and Applied Statistics, University of Wollongong, \\ Wollongong, NSW 2522 Australia \\ (2) Department of Mathematics and Statistics, Sultan Qaboos University, \\ Sultanate of Oman \\ (3) School of Physical, Environmental and Mathematical Science, UNSW at ADFA, \\ Canberra, ACT 2600, Australia
}

\begin{abstract}
In this research we analyze the steady-state operation of a continuous flow bioreactor, with or without recycle, and an idealised, or non-idealised, continuous flow membrane reactor. The model extends to include a fixed bed reactor where a fraction of the biomass is detached by the flow. The reaction is assumed to be governed by Tessier growth kinetics. We show that a flow reactor with idealised recycle has the same performance as an idealised membrane reactor and that the performance of a non-idealised membrane reactor is identical to an appropriately defined continuous flow bioreactor with non-idealised recycle. The performance of all three reactor types can therefore be obtained by analyzing a flow reactor with recycle. The steady-states of the recycle model are found and their stability determined as a function of the residence time. The performance of the reactor at large residence times is obtained.
\end{abstract}

Keywords bioreactor; kinetics; membrane reactor; modelling; stirred tank.

\section{Introduction}

A continuous flow bioreactor is a well-stirred vessel containing microorganisms $(X)$ through which a substrate $(S)$ flows at a continuous rate. The microorganisms grow in the vessel through the consumption of the substrate to produce more microorganisms and products $(P)$. The products will typically contain carbon dioxide, water and other species, including biological compounds, specific to the process under consideration. The nature of

\footnotetext{
${ }^{*}$ Corresponding author. School of Mathematics and Applied Statistics, University of Wollongong, Wollongong, NSW 2522 Australia. Telephone: +61-2-4221-440. Fax: +61-2-4221-4845. Email: nelsonm@member.ams.org.
} 
these products is unimportant in this study, as they do not affect the results presented here. Unused substrate, microorganisms, and the product flow out of the reactor.

In a bioreactor with recycle the effluent emerging from the reactor is fed into a settling unit. Microorganisms settle to the bottom of the tank, from where they are recycled into the reactor vessel. As a consequence of settling, the concentration of microorganisms leaving the settling unit in the recycle stream is higher than that entering it from the biological reactor. The settling of the microorganisms greatly reduces their concentration in the effluent leaving the settling unit, producing a cleaner effluent stream. Recycle enables a higher concentration of microorganisms to be maintained in the bioreactor, which allows the reactor to run at much greater flow-rates and increases its efficiency. This process is illustrated in figure 1.

Bioreactors sometimes employ a permeable membrane, such as a microfiltration membrane, to physically retain microorganisms inside the reactor. The higher concentrations of microorganisms obtained leads to greater substrate removal, allowing for a more rapid and efficient process. This is important in processes where the aim is to reduce the substrate concentration in the effluent. Membrane reactors are also finding increasing use in many practical applications across the fields of bioprocessing engineering and chemical engineering.

In this paper we analyze the steady-state behaviour of a biological process in both a bioreactor and a membrane reactor as a function of the residence time. The biochemical model is based upon Tessier growth rate kinetics [22] with a maintenance requirement and death of the microorganisms. In section 1.1 we outline experimental investigations where Tessier kinetics have been found to describe experimental observations. Neumann \& Gujer [15] have shown that the use of an incorrect kinetic model leads to an under-estimation of uncertainty when applying regression type analysis. In light of this finding, and in view of the number of studies listed in section 1.1, we believe that it is opportune to comprehensively study the behaviour of biological systems obeying Tessier kinetics.

The objectives of the current paper are:

- to provide a more detailed investigation of the steady-state behaviour of this process model than previously undertaken;

- to extend the process model to include recycle;

- to consider the restriction of the process to an idealised membrane reactor.

Equations (1) \& (2) are a standard model for a well-stirred reactor with recycle $(\beta=1, \gamma \neq 0)$ and without recycle $(\beta=1, \gamma=0)$, c.f. [19, pages $248 \& 493]$. Our approach to defining an idealised reactor membrane bioreactor model $(\beta=\gamma=0)$ was previously used by Yoon et al [25] and Wang et al [23]. As noted in section 2.1 the case $(0<\beta<1, \gamma=0)$ represents both a fixed-bed biological process with biomass detachment and a perfusion bioreactor incorporating a cell bleed. Thus our model equations unifies a number of standard reactor models as special cases. However, what is innovative in our paper is not the generalised reactor framework but the use of Tessier growth-rate kinetics instead of the more usually studied Monod kinetics. 
Our analysis provides the baseline for investigations into the performance improvement that can be achieved in such systems through the use of reactor cascades [14].

\subsection{Tessier growth kinetics}

It is not possible to provide a comprehensive overview of biological systems that have been found to obey Tessier kinetics. We limit ourselves to outlining a few investigations.

Schulze \& Lipe [18] carried out on experiments on Escherichia coli growing upon glucose in both a batch reactor and a continuously stirred reactor. Tessier kinetics were found to better describe the behaviour of the system than Monod kinetics. These authors used a basic reactor model without recycle, $\beta=1$ and $\gamma=0$ in equation (2), and assumed that there is no death of microorganisms, $k_{d}=0$ in equation (2). They found the no-washout steady-state solutions for the cases when there is/is no maintenance requirement, $m_{s}=0$ in equation (1). They found better agreement between theory and experimental results for the model including a maintenance requirement.

Sönmezişik et al [21] showed that a double substrate model with both Tessier and Moser growth kinetics represented the experimental data for the growth of Sulfolobus solfataricus, a thermophilic sulfur-removing archeabacterium with possible use in the microbial desulfurization of coal, reasonably well. The Tessier and Moser growth kinetics represented growth on D-glucose (carbon) and ammonium sulfate (nitrogen) respectively.

McHenry and Werker [11] showed that the Tessier growth model was the most suitable to characterize bioactivity in treatment wetlands. The main objective of this work was to characterize and calibrate enzyme activity and reaction kinetics using activated sludge to monitor the performance of treatment wetlands.

Zenaitis et al [28] investigated the kinetics of the biological treatment of log yard run-off. The biomass used in the study was obtained from the recycle line of a full-scale activated sludge treatment system. Good fits to experimental data were obtained using both the Monod and the Tessier growth rate laws.

Yurt et al [27] investigated the growth of Leptothrix discophora SP-6, a manganese- and iron-oxidizing sheathed bacteria, in a chemostat under conditions in which pyruvate and oxygen were rate limiting substrates. A double substrate model for the specific growth rate using Monod growth kinetics for pyruvate and Tessier growth kinetics for oxygen showed the best correlation with experimental data. In [26] Yurt et al investigated the growth of Leptothrix discophora SP-6 in biofilms under conditions in which dissolved oxygen was the rate limiting substrate. It was found that Monod kinetics fitted the experimental data marginally better than Tessier kinetics.

Beyenal et al [3] found that a Tessier growth expression based upon a dual-substrate model, dissolved oxygen and glucose, had good agreement with experimental chemostat data describing the growth kinetics of Pseudomonas aeruginosa (a microbial that is often used in biofilm studies and for modelling biofilm accumulation).

Guisasola et al [6] investigated nitrification by ammonia oxidizing biomass under conditions of inorganic carbon 
limitation. Oxygen uptake kinetics were successfully modelled using Monod, Tessier and sigmoidal kinetics with the best fit being sigmoidal.

Sivaprakasam et al [20] investigated the kinetics of the halo-tolerant bacteria Pseudomonas aeruginosa, isolated from tannery saline wastewater, growing upon glucose. The Tessier model was found to give a better fit to experimental data than the Monod model.

Annuar et al [1] presented a kinetic model for the growth and biosynthesis of medium-chain length poly-(3hydroxyalkanoates) in Pseudomonas putida using saponified palm kernel oil as the carbon source and ammonium as the limiting nutrient. The growth of the microorganism is well-described using a Tessier model that was adopted to take into account the inhibitory effect of the substrate at high concentrations.

Neumann \& Gujer [15] examined how parameter estimates are affected by model structure uncertainty. They did this by fitting a Monod model to synthetic data generated by a reference system using a Tessier kinetics and a known error process. Their main conclusion is that using the incorrect model leads to an under-estimation of uncertainty when applying regression type analysis.

Steady-state operation of continuous bioreactors does not necessarily give optimum reactor performance. Superior reactor performance can sometimes be achieved by periodic variation of a process variable. A number of authors have investigated the effect of periodic forcing upon systems governed by Tessier kinetics [9, 10, 24].

Liu \& Wu [9] showed that the performance of a bioreactor, measured at its optimal steady-state, can not be improved when the flow rate is forced sinusoidally. This holds for Monod, Moser, Tessier and Andrew growth models. Liu et al [10] have shown that it is possible to distinguish between Monod, Moser, Tessier and Contois kinetic models though frequency response analysis when the flow-rate is varied sinusoidally. Wu et al [24] compared the biomass production of a continuous bioreactor with a cyclic feed concentration against that produced under optimal steady-state operation. They considered Monod, Moser, Tessier and Andrew growth models. For Tessier growth, periodic operation can not improve the reactor performance when the yield coefficient is constant.

\section{Equations}

In this section we write down the model equations for the concentration of microorganisms and substrate within a well-stirred, well aerated, bioreactor and a membrane reactor. The assumption that the reactor is well-stirred means that the substrate is instantaneously and homogeneously mixed with the reactor contents. The assumption that the reactor is well aerated means that oxygen is not a rate-limiting substance. It is also assumed that the flow through the bioreactor is sufficiently fast that cell-growth does not occur on the walls of the reactors and that operating conditions are such that the $\mathrm{pH}$, temperature and other thermodynamic variables are automatically controlled so as to remain constant and spatially uniform.

The equation for the concentration of the substrate, equation (1), includes a maintenance energy term $-m_{s} X$. 
This recognizes that not all of the energy generated by substrate consumption is used for growth. Other functions, such as maintaining cell integrity and supplying the energy for cellular processes, require energy maintenance. The use of maintenance energy in bioreactor models was popularized by Pirt [17], although it had been introduced earlier by Schulze and Lipe [18].

The equation for the concentration of the microorganisms, equation (2), includes a term $-k_{d} X$ which represents a combination of first-order processes. These include endogenous respiration, predation, and cell death and lysis $[16]$.

There are two important assumptions in the process model. These are that the settling unit does not separate the substrate and that utilization of the substrate only occurs in the reactor, i.e. there is no reaction in either the settling tank or the return line. It follows from these assumptions that, although substrate is recycled, the recycling of substrate does not appear in equation (1) [19, pages 248, 493]. It should be further noted that other limiting factors, such as settling problems or membrane fouling, are ignored in the model.

\subsection{The dimensional model}

The model equations are

$$
\begin{aligned}
V \frac{\mathrm{d} S}{\mathrm{~d} t} & =F\left(S_{0}-S\right)-\frac{\mu(S)}{\alpha} V X-V m_{s} X \\
V \frac{\mathrm{d} X}{\mathrm{~d} t} & =\beta F\left(X_{0}-X\right)+\gamma R F(\mathcal{C}-1) X+V X \mu(S)-V k_{d} X
\end{aligned}
$$

Specific growth rate

$$
\mu(S)=\mu_{m}\left(1-\exp \left[-\frac{S}{K_{s}}\right]\right)
$$

Residence time

$$
\tau=\frac{V}{F}
$$

where $\mathcal{C}, \mathcal{C}>1$, is the recycle concentration factor $(-), F$ is the flowrate through the bioreactor $\left(\mathrm{dm}^{3} \mathrm{hr}^{-1}\right)$, $K_{s}$ is the Monod constant $\left(\mathrm{g} \mathrm{dm}^{-3}\right), R$ is the recycle ratio based on volumetric flow rates (-), $S$ is the substrate concentration within the bioreactor $\left(\mathrm{g} \mathrm{dm}^{-3}\right), S_{0}$ is the concentration of substrate flowing into the reactor $\left(\mathrm{g} \mathrm{dm}^{-3}\right), V$ is the volume of the bioreactor, $X$ is the concentration of cell-mass $\left(\mathrm{g} \mathrm{dm}^{-3}\right), X_{0}$ is the concentration of cell-mass flowing into the reactor $\left(\mathrm{g} \mathrm{dm}^{-3}\right), k_{d}$ is the death coefficient $\left(\mathrm{hr}^{-1}\right), m_{s}$ is the maintenance coefficient $\mathrm{hr}^{-1}, t$ is time $\left(\mathrm{hr}^{-1}\right), \alpha$ is the yield factor $(-), \mu$ is the specific growth rate model, $\mu_{m}$ is the maximum specific growth rate $\left(\mathrm{hr}^{-1}\right)$, and $\tau$ is the residence time (hr).

The parameters $\beta$ and $\gamma$ define the reactor model. The choice $\beta=\gamma=1$ gives a continuous flow reactor. The choice $\beta=\gamma=0$ gives an idealised membrane reactor, in which all of the microorganisms is constrained to remain in the reactor vessel. Although highly simplified, this approach to modelling membrane bioreactors was previously used by Yoon et al [25] and Wang et al [23]. In both cases a submerged membrane bioreactor 
configuration was considered. The model developed by Wang et al [23] was used to analyse data from a benchscale study The choice $0<\beta<1$ and $\gamma=0$ gives a non-idealised membrane reactor, in which some of the microorganisms leave the reactor vessel in the effluent stream. For a non-idealised membrane reactor to be operationally effective we require $0<\beta \ll 1$. The case $0<\beta<1$ and $\gamma=0$ with $X_{0}=0$ represents two other reactor models. Firstly, it models a fixed-bed biological process in which $\beta$ is the fraction of the biomass that is detached from the reactor by the flow [2, page 428]. Secondly, it models the perfusion bioreactor incorporating a cell bleed considered in $[4,5]$.

In equation (2) the term $\mathcal{C} X$ is the biomass concentration in the flow leaving the separating unit. The value of the concentrating factor depends upon the design and operation of the settling unit. It is also highly dependent on sludge properties such as settling, thickening and compressibility behaviour. A mass balance around the settling units shows that the maximum value of the concentrating factor is given by

$$
\mathcal{C}_{\max }=1+\frac{1}{R}
$$

Thus the maximum value of the product $R(\mathcal{C}-1)$ is

$$
[R(\mathcal{C}-1)]_{\max }=1 .
$$

This is the simplest possible model for recycle from a settling unit.

For a specific wastewater, a given biological community and a particular set of environmental conditions the parameters $K_{s}, k_{d}, m_{s}, \alpha$ and $\mu_{\max }$ are fixed. The parameters that can be varied are $S_{0}, X_{0}$ and $\tau$. The main experimental control parameter is the residence time, given in equation (4).

\subsubsection{The dimensionless model}

By introducing dimensionless variables for the substrate concentration $\left[S^{*}=S / K_{s}\right]$, the cell mass concentration $\left[X^{*}=X /\left(\alpha K_{s}\right)\right]$ and time $\left[t^{*}=\mu_{m} t\right]$ the dimensional model, equation (1) \& (2), can be written in the dimensionless form

$$
\begin{aligned}
\frac{\mathrm{d} S^{*}}{\mathrm{~d} t^{*}} & =\frac{1}{\tau^{*}}\left(S_{0}^{*}-S^{*}\right)-\left(1-\exp \left[-S^{*}\right]\right) X^{*}-m_{s}^{*} X, \\
\frac{\mathrm{d} X^{*}}{\mathrm{~d} t^{*}} & =\beta \frac{1}{\tau^{*}}\left(X_{0}^{*}-X^{*}\right)+\gamma \frac{R^{*}}{\tau^{*}} X^{*}+\left(1-\exp \left[-S^{*}\right]\right) X^{*}-k_{d}^{*} X^{*},
\end{aligned}
$$

where the parameter groups are: the effective recycle parameter $\left[R^{*}=(\mathcal{C}-1) R\right]$, the dimensionless substrate concentration in the feed $\left[S_{0}^{*}=S_{0} / K_{s}\right]$, the dimensionless biomass concentration in the feed $\left[X_{0}^{*}=X_{0} /\left(\alpha K_{s}\right)\right]$, the dimensionless decay rate $\left[k_{d}^{*}=k_{d} / \mu_{m}\right]$, the dimensionless maintenance energy $\left[m_{s}^{*}=\alpha m_{s} / \mu_{m}\right]$ and the dimensionless residence time $\left[\tau^{*}=V \mu_{m} / F\right]$, All parameters in the model are strictly non-negative.

From now on we assume that the growth medium fed into the bioreactor is sterile, i.e. there are no microorganisms in the influent $\left(X_{0}=X_{0}^{*}=0\right)$, and that $S_{0}^{*}>0$. From equation (5) it follows that the maximum value 
of the effective recycle parameter is $R^{*}=1$. The cases $R^{*}=1$ and $0<R^{*}<1$ with $\beta=\gamma=1$ define a flow reactor with idealised and non-idealised recycle respectively.

With the assumption of sterile feed $\left(X_{0}^{*}=0\right)$ it follows from equation $(7)$ that a flow reactor model with idealised recycle $\left(\beta=\gamma=R^{*}=1\right)$ is identical to the idealised membrane reactor model $(\beta=\gamma=0)$. Furthermore, with the substitution $\beta=1-R^{*}$ the equations for a non-idealised membrane reactor $(0<\beta<1, \gamma=0)$ are identical to those of a flow reactor with non-idealised recycle $\left(\gamma=\beta=1, R^{*}>0\right)$. This statement is independent of the form used for the specific growth rate expression and was first made in [13].

\section{Results}

In this section we analyze the flow-reactor with recycle $\left(\beta=\gamma=1,0 \leq R^{*} \leq 1\right)$ as we have shown that the results for a membrane reactor $(\beta=\gamma=0)$ immediately follow with the substitution $R^{*}=1-\beta$.

In section 3.1 the steady-state solution branches are given and the condition for the no-washout solution branch to be physically meaningful is identified. In section 3.2 the stability of the steady-state solutions is determined. In section 3.3 asymptotic solutions for large residence times are stated. In section 3.4 we compare the effluent concentration leaving an idealised membrane reactor to that leaving a conventional sewage sludge digester.

\subsection{Steady-state solution branches}

The steady-state solutions are given by

$$
\begin{aligned}
& \text { Washout branch } \\
\left(S^{*}, X^{*}\right) & =\left(S_{0}^{*}, 0\right), \\
& \text { No-washout branch } \\
\left(S^{*}, X^{*}\right) & =\left(\hat{S^{*}}, \frac{S_{0}^{*}-\hat{S}^{*}}{1-R^{*}+\left(k_{d}^{*}+m_{s}^{*}\right) \tau^{*}}\right), \\
\hat{S}^{*} & =\ln \left[\frac{\tau^{*}}{R^{*}-1+\left(1-k_{d}^{*}\right) \tau^{*}}\right]
\end{aligned}
$$

The cases $R^{*}=0,0<R^{*}<1$, and $R^{*}=1$ represent a flow reactor without recycle, a flow reactor with non-idealised recycle, and a flow reactor with idealised recycle respectively. The no-washout branch is only physically meaningful when the substrate and cell-mass concentrations are positive $\left(S^{*}>0, X^{*}>0\right)$.

When $0 \leq R^{*}<1$ the no-washout branch is physically meaningful when

$$
\begin{gathered}
\tau^{*}>\frac{1-R^{*}}{1-e^{-S_{0}^{*}-k_{d}^{*}},} \\
0 \leq k_{d}^{*}<1-e^{-S_{0}^{*}} .
\end{gathered}
$$


When $R^{*}=1$ the no-washout branch is physically meaningful when

$$
\begin{aligned}
\left(k_{d}^{*}+m_{s}^{*}\right) \tau^{*} & >0 \\
0 & <k_{d}^{*}<1-e^{-S_{0}^{*}}
\end{aligned}
$$

Differentiating equation (10) we obtain

$$
\frac{\mathrm{d} S^{*}}{\mathrm{~d} R^{*}}=\frac{-1}{\left[R^{*}-1+\left(1-k_{d}^{*}\right) \tau^{*}\right]}<0,
$$

as when the no-washout solution branch is physically meaningful we have $R^{*}-1+\left(1-k_{d}^{*}\right) \tau^{*}>0$. Thus the substrate concentration is a decreasing function of the effective recycle parameter. This mathematical result is well known experimentally - recycle reactors reduce the effluent concentration which is the effect of making previously uneconomical treatment regimes economical. It is straightforward to show that the substrate concentration leaving the reactor is a monotonic decreasing function of the residence time, i.e. the lowest effluent concentration is obtained at an infinite residence time.

The residence time which maximizes the microorganism concentration in the reactor can be easily found by differentiating equations (9) \& (10) and solving the resulting transcendental equation numerically. However, the resulting expression does not lead to any analytical insights.

For a flow reactor with idealised recycle, or an idealised membrane reactor, $\left(R^{*}=1\right)$ the substrate and biomass concentrations are given by

$$
\begin{aligned}
& S^{*}\left(R^{*}=1\right)=\ln \left[\frac{1}{1-k_{d}^{*}}\right] . \\
& X^{*}\left(R^{*}=1\right)=\frac{S_{0}^{*}+\ln \left(1-k_{d}^{*}\right)}{\left(k_{d}^{*}+m_{s}^{*}\right) \tau^{*}} .
\end{aligned}
$$

The substrate concentration is independent of the residence time. The microorganism concentration grows unbounded in size as the residence time approaches zero which is physically meaningless. Thus the model can not apply at sufficiently low residence times for an idealised membrane reactor.

\subsection{Stability of the steady-state solutions}

The Jacobian matrix is given by

$$
J\left(S^{*}, X^{*}\right)=\left(\begin{array}{cc}
-\frac{1}{\tau^{*}}-X^{*} e^{-S^{*}} & -\left(1-e^{-S^{*}}\right)-m_{s}^{*} \\
X^{*} e^{-S^{*}} & \frac{R^{*}-1}{\tau^{*}}+\left(1-e^{-S^{*}}\right)-k_{d}^{*}
\end{array}\right) .
$$

The Jacobian matrix evaluated at the washout steady-state solution is given by

$$
J\left(S_{0}^{*}, 0\right)=\left(\begin{array}{cc}
-\frac{1}{\tau^{*}} & -\left(1-e^{-S_{0}^{*}}\right)-m_{s}^{*} \\
0 & \frac{R^{*}-1}{\tau^{*}}+\left(1-e^{-S_{0}^{*}}\right)-k_{d}^{*}
\end{array}\right) .
$$

The eigenvalues of this matrix are

$$
\begin{aligned}
& \lambda_{1}=-\frac{1}{\tau^{*}}<0, \\
& \lambda_{2}=\frac{R^{*}-1}{\tau^{*}}+\left(1-e^{-S_{0}^{*}}\right)-k_{d}^{*} .
\end{aligned}
$$


It follows that the washout branch is always stable if

$$
k_{d}^{*}>1-e^{-S_{0}^{*}} .
$$

In particular, the washout steady-state is always stable when

$$
\begin{aligned}
k_{d}^{*} & \geq 1, \\
\Rightarrow \frac{k_{d}}{\mu_{m}} & \geq 1, \\
\Rightarrow k_{d} & \geq \mu_{m} .
\end{aligned}
$$

This makes physical sense because it implies that the washout steady-state will always be stable if the death rate is greater than, or equal to, the maximum growth rate.

If $k_{d}^{*}<1-e^{-s_{0}^{*}}$ then the washout steady-state is stable provided

$$
\tau^{*}<\frac{1-R^{*}}{\left(1-e^{-S_{0}^{*}}\right)-k_{d}^{*}} .
$$

The Jacobian matrix for the no-washout branch is given by

$$
J\left(S^{*}, X^{*}\right)=\left(\begin{array}{cc}
-\frac{1}{\tau^{*}}-X^{*} e^{-S^{*}} & -\left(1-e^{-S^{*}}\right)-m_{s}^{*} \\
X^{*} e^{-S^{*}} & 0
\end{array}\right) .
$$

This solution branch is stable if $\operatorname{det} J>0$ and $\operatorname{trace} J<0$. We have

$$
\begin{aligned}
\operatorname{det} J & =\left(1-e^{-S^{*}}+m_{s}^{*}\right) X^{*} e^{-S^{*}} \\
\operatorname{trace} J & =-\frac{1}{\tau^{*}}-X^{*} e^{-S^{*}}
\end{aligned}
$$

It therefore follows that $\operatorname{det} J>0$ and trace $J<0$ whenever $X^{*}>0$ and $S^{*}>0$. Thus the no-washout branch is stable whenever it is physically meaningful.

A transcritical bifurcation occurs, as the residence time is varied, when

$$
\tau^{*}=\tau_{\mathrm{tr}}^{*}=\frac{1-R^{*}}{1-e^{-S_{0}^{*}-k_{d}^{*}}} .
$$

At this value of the residence time the no-washout solution branch and the washout solution branch intersect at the point

$$
\left(S^{*}, X^{*}, \tau^{*}\right)=\left(1,0, \tau_{\mathrm{tr}}^{*}\right)
$$

The value of the residence time represents the maximum residence time at which the treatment process fails. At lower residence times microorganisms are removed from the system at a rate greater than their maximum growth rate, resulting in process failure. At residence times lower (higher) than the transcritical value the washout (no-washout) solution is the only stable solution. Schulze \& Lipe [18] did not determine conditions for the stability of the no-washout branch. Equation (11) shows that the washout condition is a linearly decreasing function of the effective recycle parameter, reaching a zero value for a flow reactor with idealised recycle (or for 
an idealised membrane reactor). Thus recycle allows the process to be operate at lower residence times than would otherwise be the case - allowing a greater throughput of effluent.

Steady-state diagrams showing how the dimensionless cell mass concentration $\left(X^{*}\right)$ and the dimensionless substrate concentration $\left(S^{*}\right)$ vary as the dimensionless residence time is varied are shown in Figure 2 - only physically meaningful solutions have been plotted. That is, in Figures 2 (a) and (b) the stable solution for sufficiently low residence times $\left(\tau^{*}<\tau_{\mathrm{tr}}^{*}\right)$ are the lines $S^{*}=S_{0}^{*}=1$ and $X^{*}=0$ respectively.

\subsection{Large residence time approximations}

At large residence times we have the approximations

$$
\begin{aligned}
& S^{*} \approx \ln \left(\frac{1}{1-k_{d}^{*}}\right)+\frac{\left(1-R^{*}\right)}{\left(1-k_{d}^{*}\right)} \cdot \frac{1}{\tau^{*}}+O\left(\frac{1}{\tau^{*^{2}}}\right), \\
& X^{*} \approx \frac{S_{0}^{*}+\ln \left(1-k_{d}^{*}\right)}{\left(k_{d}^{*}+m_{s}^{*}\right)} \cdot \frac{1}{\tau^{*}}+O\left(\frac{1}{\tau^{*^{2}}}\right) .
\end{aligned}
$$

At large residence times the microorganism concentration is, to leading order, independent of the value of the effective recycle factor $\left(R^{*}\right)$, i.e. it is independent of the reactor configuration. In these formulae the case $k_{d}^{*}=1$ is not allowed, as when this is true the no-washout branch is not physically meaningful; see section 3.1 .

Consider a well-stirred flow reactor that is being used to reduce the concentration of a pollutant in an industrial wastewater or slurry. Equation (12) shows that at high residence times there is a limiting concentration, $S^{*}=\ln \left(\frac{1}{1-k_{d}^{*}}\right)$, below which the effluent concentration can not be reduced. Note that in a flow reactor with non-idealised recycle the limiting concentration is only reached at an infinite residence time. In a flow reactor with idealised recycle, or an idealised membrane reactor, the same performance can be achieved at any finite residence time. The same conclusion holds when Monod kinetics are used [13]. It does not hold when the specific growth rate law is a function of the concentration of both the substrate and biomass, as us this case for Contois kinetics [12].

\subsection{Effluent concentration}

One attraction of an idealised membrane reactor $\left(R^{*}=1\right)$ is that all the microorganisms remain in the bioreactor, leading to a higher microorganism concentration than in either a conventional bioreactor $\left(R^{*}=0\right)$ or a nonidealised recycle reactor $\left(0<R^{*}<1\right)$. This leads to a lower effluent concentration leading the reactor. Comparing the effluent concentration leaving an idealised membrane reactor to that leaving a conventional sewage sludge digester we have

$$
\frac{S^{*}\left(R^{*}=1\right)}{S^{*}\left(R^{*}=0\right)}=\left\{\begin{array}{lll}
\frac{-\ln \left(1-k_{d}^{*}\right)}{S_{0}^{*}} & \text { if } & 0<\tau^{*} \leq \tau_{\mathrm{tr}}^{*} \\
\frac{-\ln \left(1-k_{d}^{*}\right)}{\ln \left(\tau^{*}\right)-\ln \left[\left(1-k_{d}^{*}\right) \tau^{*}-1\right]} & \text { if } & \tau_{\mathrm{tr}}^{*}<\tau^{*}
\end{array}\right.
$$

Thus the difference between the two reactor configurations decreases to zero as the residence time increases to infinity. Note that in this formula we require $k_{d}^{*}>0$. (As otherwise there is no steady-state solution for the 
idealised recycle reactor). The maximum difference in effluent concentration occurs for residence times in which the stable solution in the conventional reactor is the washout branch.

\section{Discussion}

There are a number of definitions which are used to characterize the steady-state performance of a continuous flow bioreactor processing industrial wastewaters [8]. The results stated in this section only apply when the no-washout branch is physically meaningful. We require

$$
\tau^{*}>\tau_{\mathrm{tr}}^{*}=\frac{1-R^{*}}{1-e^{-S_{0}^{*}-k_{d}^{*}}} .
$$

The dimensionless specific utilization $(\mathcal{U})$, which is also known as the process loading factor, the substrate removal velocity or the food to microorganism ratio, is the rate of substrate utilization per unit mass of microorganisms and is defined by

$$
\mathcal{U}=\frac{S_{0}^{*}-S^{*}}{X^{*}} \cdot \frac{1}{\tau^{*}}
$$

Using equations (9) and (10) we obtain the specific utilization as

$$
\mathcal{U}=\left(k_{d}^{*}+m_{s}^{*}\right)+\frac{1-R^{*}}{\tau^{*}} .
$$

Hence the dimensionless specific utilization is a linearly decreasing function of the effective recycle parameter and is independent of the feed concentration $\left(S_{0}^{*}\right)$. The expression for the specific utilization (14) holds for any choice of growth rate function in equation (3) of the form $\mu(X)$ or $\mu(S, X)$.

The dimensionless treatment, or process, efficiency $(\mathcal{E})$ is defined by

$$
\mathcal{E}=100 \cdot \frac{S_{0}^{*}-S^{*}}{S_{0}^{*}} .
$$

Note from this definition it is clear that along the no-washout branch, where $S^{*}<S_{0}^{*}$, that the efficiency is positive. Using equation (9) we obtain the efficiency as

$$
\mathcal{E}=100\left\{1-\frac{1}{S_{0}^{*}} \ln \left[\frac{\tau^{*}}{R^{*}-1+\left(1-k_{d}^{*}\right) \tau^{*}}\right]\right\} .
$$

At large residence times this becomes

$$
\mathcal{E} \approx 100\left[1+\frac{\ln \left(1-k_{d}^{*}\right)}{S_{0}^{*}}-\frac{\left(1-R^{*}\right)}{\left(1-k_{d}^{*}\right) S_{0}^{*}} \cdot \frac{1}{\tau^{*}}+O\left(\frac{1}{\tau^{*}}\right)^{2}\right]
$$

The maximum efficiency is obtained either in the limit of infinite residence time, for a flow reactor with nonidealised recycle, or for a finite time, for an idealised membrane reactor, and is given by

$$
\mathcal{E}_{\max }=100\left[1+\frac{1}{S_{0}^{*}} \ln \left(1-k_{d}^{*}\right)\right] .
$$


The efficiency and the maximum efficiency both increase as the loading of the effluent increases. Furthermore, the efficiency is an increasing function of the effective recycle parameter.

The dimensionless rate of waste treatment is defined by

$$
\mathcal{W}=\frac{S_{0}^{*}-S^{*}}{\tau^{*}}
$$

Note from this definition it is clear that along the no-washout branch, where $S^{*}<S_{0}^{*}$, that the rate of waste treatment is positive. Using equation (9) we obtain the rate of waste treatment as

$$
\mathcal{W}=\left\{S_{0}^{*}-\ln \left[\frac{\tau^{*}}{R^{*}-1+\left(1-k_{d}^{*}\right) \tau^{*}}\right]\right\} \cdot \frac{1}{\tau^{*}} .
$$

Thus the rate of waste treatment increases linearly as the loading of the effluent increases. Furthermore, the rate of waste treatment is an increasing function of the effective recycle parameter. For a fixed residence time the maximum rate of waste treatment is obtained for a membrane reactor and is given by

$$
\mathcal{W}\left(R^{*}=1\right)=\frac{S_{0}^{*}+\ln \left(1-k_{d}^{*}\right)}{\tau^{*}}
$$

At large residence times the rate of waste treatment is given by

$$
\mathcal{W} \approx\left\{S_{0}^{*}+\ln \left(1-k_{d}^{*}\right)\right\} \cdot \frac{1}{\tau^{*}}+O\left(\frac{1}{\tau^{*}}\right)^{2}
$$

Other ways of measuring the performance of a continuous flow reactor include the dimensionless yield $(\mathcal{Y})$ and the effective dimensionless yield $\left(\mathcal{Y}_{e}\right)[7]$ defined by

$$
\begin{aligned}
\mathcal{Y} & =\frac{X^{*}}{S_{0}^{*}-S^{*}}, \\
\mathcal{Y}_{e} & =\frac{X^{*}}{S_{0}^{*}} .
\end{aligned}
$$

These definitions are more appropriate for processes in which microorganisms are being cultivated rather than to characterize the operation of waste treatment facilities. Substituting the steady-state values for the substrate and microorganism concentrations, equations (9) and (10), we obtain

$$
\begin{aligned}
\mathcal{Y} & =\frac{1}{1-R^{*}+\left(k_{d}^{*}+m_{s}^{*}\right) \tau^{*}}, \\
\mathcal{Y}_{e} & =\frac{1}{S_{0}^{*}} \cdot \frac{S_{0}^{*}-\ln \left[\frac{\tau^{*}}{R^{*}-1+\left(1-k_{d}^{*}\right) \tau^{*}}\right]}{\left(k_{d}^{*}+m_{s}^{*}\right) \tau^{*}+1-R^{*}} .
\end{aligned}
$$

The expression for the yield holds for any choice of the growth-rate function of the form $\mu(S)$ or $\mu(S, X)$. It is a decreasing function of both the residence time and the effective recycle parameter and does not depend upon the feed concentration $\left(S_{0}^{*}\right)$. The effective yield coefficient is maximised at the same residence time that maximizes the microorganism concentration. At large values of the residence time the effective yield is given by

$$
\mathcal{Y}_{e} \approx \frac{S_{0}^{*}+\ln \left(1-k_{d}^{*}\right)}{\left(k_{d}^{*}+m_{s}^{*}\right) S_{0}^{*}} \cdot \frac{1}{\tau^{*}}+O\left(\frac{1}{\tau^{*^{2}}}\right) \text {. }
$$


For large values of the feed concentration this becomes

$$
\mathcal{Y}_{e} \approx \frac{1}{k_{d}^{*}+m_{s}^{*}} \cdot \frac{1}{\tau^{*}}
$$

In the limit of large feed concentration and large residence times the expression for the effective yield is the same for Tessier kinetics and Monod kinetics [13].

\section{Conclusion}

In this paper we have investigated a generalised reactor model for the interaction between a microorganism and a rate-controlling substrate. The reactor model contains a number of standard reactor models as special cases: fixed bed reactors, membrane reactors, a perfusion bioreactor incorporating a cell bleed, in addition to a well-stirred flow reactor, with or without recycle. The biochemical model examined here was the Tessier expression for the specific growth rate and included both a microorganism decay coefficient and a maintenance energy requirement. The use of Tessier kinetics, rather than the well-studied Monod kinetics, is justified by the number of experimental studies listed in section 1.1 for which Tessier kinetics have been found to accurately describe experimental data. The pertinence of this study is illustrated by the finding of Neumann \& Gujer [15] that the use of an incorrect kinetic model leads to an under-estimation of uncertainty when applying regression type analysis.

We showed that an idealised membrane reactor is equivalent to a well-stirred flow reactor with idealised recycle. Thus results for a well-stirred flow reactor with non-idealised recycle, and those of the other reactor types, follow from those of a flow reactor with idealised recycle after a minor re-parameterization of the model.

We considered an extension of the basic biochemical model to include death of the microorganisms and a maintenance energy requirement. Although the application of Tessier kinetics to biological kinetics has a long history, the performance of a well-stirred bioreactor with microorganism death, maintenance energy and recycle has not been reported in the literature. The steady-state solutions of the model were found and their stability determined as a function of the residence time. These results are used to evaluate various performance characterizations of continuous flow bioreactors that have been proposed.

In a well-stirred bioreactor with non-idealised recycle, the effluent concentration has its minimum value at an infinite residence time. By contract, the same effluent concentration is obtained from an idealised membrane reactor operating at any finite residence time.

\section{Acknowledgements}

This work was supported by grants from the Australian Research Council (DP0559177) and Sultan Qaboos University IG/SCI/DOMS/06/07). MIN thanks the Department of Mathematics and Statistics at SQU for 
their hospitality and support during his visit. During the course of this work MIN was a Visiting Fellow in the School of Physical Environmental \& Mathematical Sciences (PEMS), UNSW@ADFA.

\section{A Symbols used}

$\mathcal{C}$

$\mathcal{E}$

$F$

$K_{s}$

$R$

$R^{*}$

$S$

$S^{*}$

$\hat{S}^{*}$

$S_{0}$

$S_{0}^{*}$

$\mathcal{U}$

V

$\mathcal{W}$

$X$

$X^{*}$

$X_{0} \quad$ Concentration of microorganisms in the feed.

$X_{0}^{*} \quad$ Dimensionless microorganism concentration in the feed.

$X_{0}^{*}=X_{0} /\left(\alpha K_{s}\right)$

$\mathcal{Y} \quad$ Dimensionless yield.

$\mathcal{Y}=\frac{X^{*}}{S_{0}^{*}-S^{*}}$

$\mathcal{Y}_{e} \quad$ Dimensionless effective yield.

$\mathcal{Y}_{e}=\frac{X^{*}}{S_{0}^{*}}$

$k_{d} \quad$ Death coefficient.
$(-)$

$(-)$

$\left(\mathrm{gdm}^{-3}\right)$

$(-)$

$(-)$

$\left(\mathrm{g} \mathrm{dm}^{-3}\right)$

$(-)$

$(一)$

$\left(\mathrm{dm}^{3}\right)$

$($-)

$\left(\mathrm{g} \mathrm{dm}^{-3}\right)$

$(-)$

$\left(\mathrm{g} \mathrm{dm}^{-3}\right)$

$(-)$

$(-)$

$(一)$

$\left(\mathrm{hr}^{-1}\right)$ 
$k_{d}^{*} \quad$ Dimensionless death coefficient.

$k_{d}^{*}=k_{d} / \mu_{m}$

$\begin{array}{ll}m_{s} & \text { Maintenance coefficient } \\ m_{s}^{*} & \text { Dimensionless maintenance coefficient. }\end{array}$

$\left(\mathrm{hr}^{-1}\right)$

$m_{s}^{*}=\alpha m_{s} / \mu_{m}$

$t$

Time.

$(-)$

Dimensionless time.

$\left(\mathrm{hr}^{-1}\right)$

$t^{*}=\mu_{m} t$

$\alpha \quad$ Yield factor.

$\beta \quad$ Reactor parameter model.

Reactor parameter model.

$(-)$

Specific growth rate model.

Maximum specific growth rate.

residence time.

Dimensionless residence time.

$\tau^{*}=V \mu_{m} / F$

$\tau_{\mathrm{tr}}^{*} \quad$ The value of the dimensionless residence time at the transcritical bifurcation. ( - )

\section{References}

[1] M.S.M. Annuar, I.K.P. Tan, S. Ibrahim, and K.B. Ramachandran. A kinetic model for growth and biosynthesis of medium-chain-length poly-(3-hydroxyalkanoates) in Pseudomonas putida. Brazilian Journal of Chemical Engineering, 25(2):217-228, 2008.

[2] O. Bernard, Z. Hadj-Sadok, D. Dochain, A. Genovesi, and J.P. Steyer. Dynamical model development and parameter identification for an anaerobic wastewater treatment process. Biotechnology and Bioengineering, $75(4): 424-438,2001$.

[3] H. Beyenal, S.N. Chen, and Z. Lewandowski. The double substrate growth kinetics of Pseudomonas aeruginosa. Enzyme and Microbial Technology, 32:92-98, 2003.

[4] J-S. Deschênes, A. Desbiens, M. Perrier, and A. Kamen. Multivariable nonlinear control of biomass and metabolite concentrations in a high-cell-density perfusion bioreactor. Industrial \& Engineering Chemistry Research, 45(26):8985-8997, 2006.

[5] J-S. Deschênes, A. Desbiens, M. Perrier, and A. Kamen. Use of cell bleed in a high cell density perfusion culture and multivariable control of biomass and metabolite concentrations. Asia-Pacific Journal of Chemical Engineering, 1:82-91, 2006.

[6] A. Guisasola, S. Petzet, J.A. Baeza, J. Carrera, and J. Lafuente. Inorganic carbon limitations on nitrification: Experimental assessment and modelling. Water Research, 41:277-286, 2007. 
[7] D. Herbert, R. Elsworth, and R.C. Telling. The continuous culture of bacteria: a theoretical and experimental study. Journal of General Microbiology, 14:601-622, 1956.

[8] A.W. Lawrence and P.L. McCarty. Unified basis for biological treatment design and operation. Journal of the Sanitary Engineering Division — ASCE, 96(SA3):757-778, 1970.

[9] H-S Liu and S-C Wu. Periodical operation of continuous bioreactor. Journal of the Chinese Institute of Chemical Engineers, 26(5):233-243, 1995.

[10] H-S Liu, S-C Wu, and S-C Lin. Theoretical analysis of frequency response in continuous bioreactor. Chemical Engineering Communications, 164:13-34, 1998.

[11] J. McHenry and A.G. Werker. Characterization of bioactivity in treatment wetlands utilising an enzymatic assay. In CSCE/EWRI of ASCE Environmental Engineering Conference, pages 1-14, 2002.

[12] M.I. Nelson, E. Balakrishnan, H.S. Sidhu, and X.D. Chen. A fundamental analysis of continuous flow bioreactor models and membrane reactor models to process industrial wastewaters. Chemical Engineering Journal, 140(1-3):521-528, 2008. dx.doi.org/10.1016/j .cej .2007.11.035.

[13] M.I. Nelson, T. Kerr, and X.D. Chen. A fundamental analysis of continuous flow bioreactor and membrane reactor models with death and maintenance included. Asia Pacific Journal of Chemical Engineering, 3:70-80, 2008. dx.doi .org/10.1002/apj . 106.

[14] M.I. Nelson and H.S. Sidhu. Evaluating the performance of a cascade of two bioreactors. Chemical Engineering Science, 61(10):3159-3166, 2006. http://dx.doi.org/10.1016/j .ces.2005.12.007.

[15] M.B. Neumann and W. Gujer. Underestimation of uncertainty in statistical regression of environmental models: Influence of model structure uncertainty. Environmental Science and Technology, 42:4037-4043, 2008 .

[16] S.G. Pavlostathis and E. Giraldo-Gomez. Kinetics of anaerobic treatment. Water Science and Technology, $24(8): 35-59,1991$.

[17] S.J. Pirt. The maintenance energy of bacteria in growing cultures. Proceeding of the Royal Society of London B, 163:224-231, 1965.

[18] K.L. Schulze and R.S. Lipe. Relationship between substrate concentration, growth rate, and respiration rate of escherichia coli in continuous culture. Archiv für Mikrobiologie, 48:1-20, 1964.

[19] M.L. Shuler and F. Kargi. Bioprocess Engineering. Prentice Hall International Series in the Physical and Chemical Engineering Sciences. Prentice Hall, New Jersey, USA, second edition, 2002.

[20] S. Sivaprakasam, S. Mahadevan, and M. Bhattacharya. Biocalorimetric and respirometric studies on metabolic activity of aerobically grown batch culture of Pseudomonas aeruginosa. Biotechnology and Bioprocess Engineering, 12:340-347, 2007. 
[21] M. Sömezişik, D. Tanyolaç, S. Şeker, and A. Tanyolaç. The double-substrate growth kinetics of Sulfolobus solfataricus a thermophilic sulfur-removing archeabacterium. Biochemical Engineering Journal, 1:243-248, 1998.

[22] G. Tessier. Les lois quantitatives de la croissance. Annales de Physiologie et de Physiochimie Biologique, 12:527-573, 1936 .

[23] Z. Wang, L. Wang, B.Z. Wang, Y.F. Jiang, and S. Liu. Bench-scale study on zero excess activated sludge production process coupled with ozonation unit in membrane bioreactor. Journal of Environmental Science and Health, Part A, 43(11):1325-1332, 2008.

[24] S-C. Wu, C-C. Lin, and H-S. Liu. Theoretical analysis of a continuous bioreactor subject to cyclic feed concentration. Journal of the Chinese Institute of Chemical Engineers, 30(2):151-160, 1999.

[25] S-H. Yoon, H-S. Kim, and I-T Yeom. The optimum operational condition of membrane bioreactor (MBR): cost estimation of aeration and sludge treatment. Water Research, 38:37-46, 2004.

[26] N. Yurt, H. Beyenal, J. Sears, and Z. Lewandowski. Quantifying selected growth parameters of Leptothrix discophora SP-6 in biofilms from oxygen concentration profiles. Chemical Engineering Science, 58:45574566, 2003.

[27] N. Yurt, J. Sears, and Z. Lewandowski. Multiple substrate growth kinetics of Leptothrix discophora SP-6. Biotechnology Progress, 18:994-1002, 2002.

[28] M.G. Zenaitis, H. Sandhu, and S.J.B. Duff. Combined biological and ozone treatment of log yard run-of. Water Research, 36:2053-2061, 2002. 


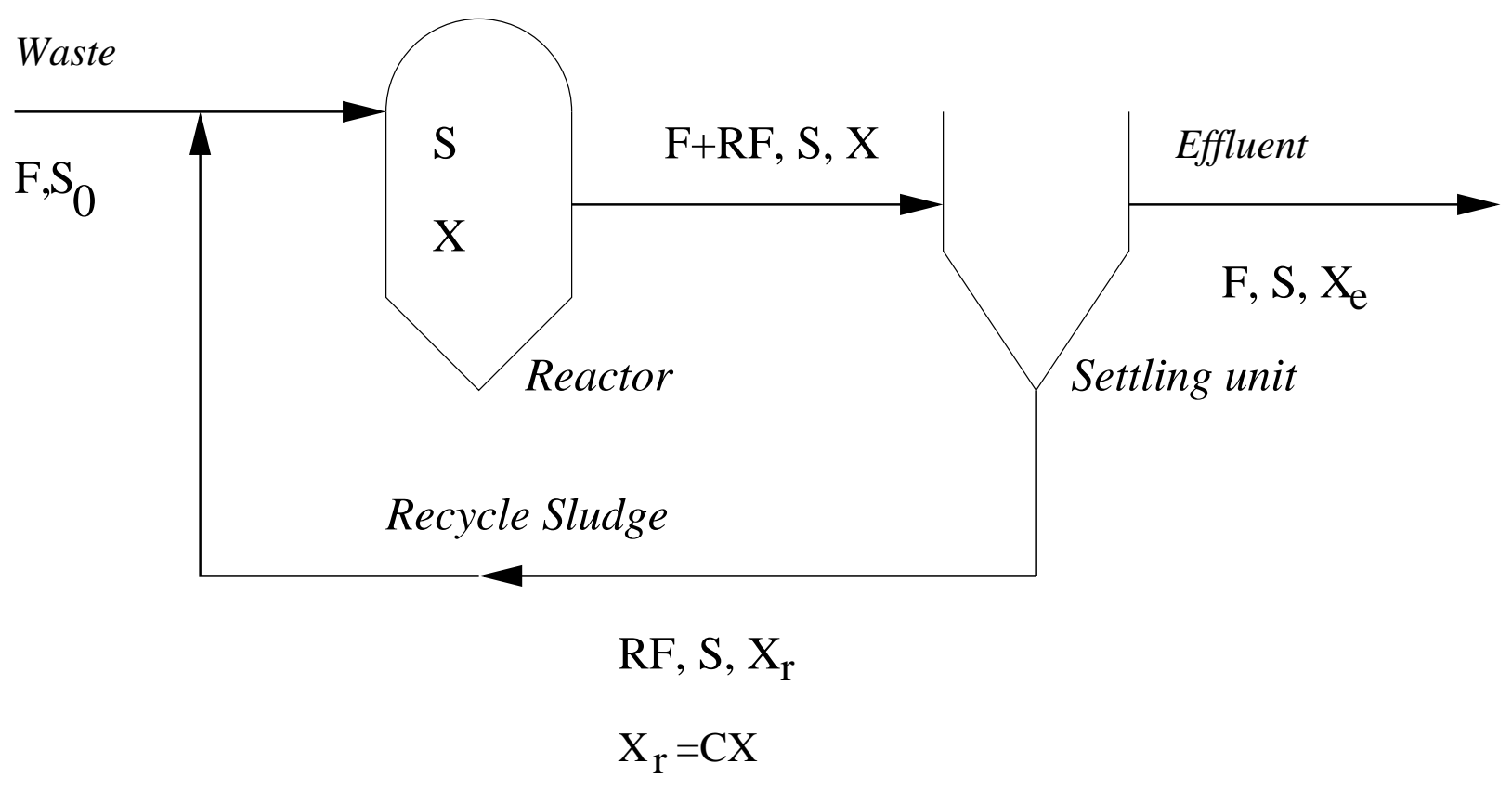

Figure 1: A bioreactor with recycle. 

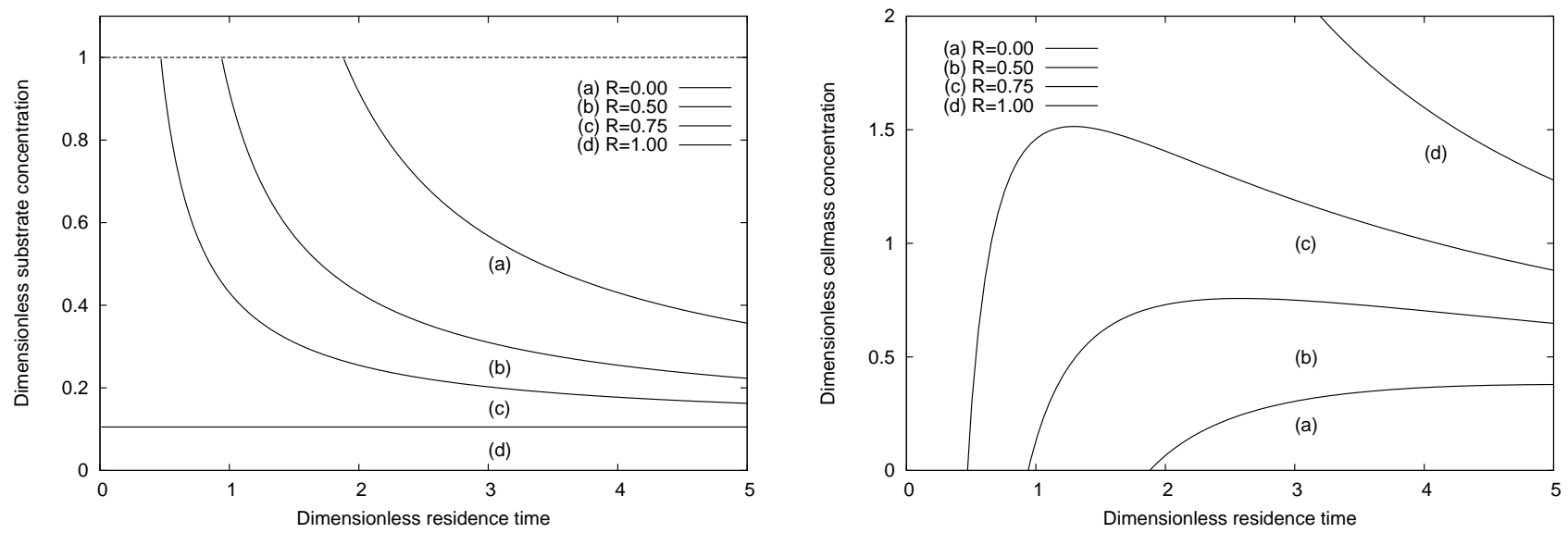

Figure 2: Steady-state diagrams showing the variation of dimensionless substrate concentration $\left(S^{*}\right)$ and cell mass concentration $\left(X^{*}\right)$ as a function of the dimensionless residence time $\left(\tau^{*}\right)$ in a well-stirred bioreactor. Parameter values: dimensionless death rate, $k_{d}^{*}=0.1$; dimensionless maintenance rate, $m_{s}^{*}=0.04$; dimensionless feed concentration, $S_{0}^{*}=1$. The value of the effective recycle parameter $\left(R^{*}\right)$ is as given. In figure (a) the dashed line $S^{*}=1$ is the washout solution branch. 